\title{
Multilayer Graphene Films Obtained by Dip Coating Technique
}

\author{
Luis Gustavo Baptista Machuno ${ }^{a}$, Alex Ricardo Oliveira ${ }^{a}$, Renan Hermógenes Furlan ${ }^{a}$, \\ Anderson Barbosa Lima ${ }^{a}$, Luis Carlos Morais ${ }^{a}$, Rogério Valentim Gelamo * \\ ${ }^{a}$ Universidade Federal do Triângulo Mineiro - UFTM, Uberaba, MG, Brazil
}

Received: April 24, 2015; Revised: July 3, 2015

\begin{abstract}
A dip coating system was projected and constructed to deposit a large range of thin films onto solid substrates. A microcontroller was used to control the deposition system. As an example of application multilayer graphene (MLG) films were deposited onto glass slices in order to obtain conductive films with relatively good light transmittance. The MLG flakes were obtained using natural graphite and isopropyl alcohol resulting in three dispersions treated in ultrasound for 30, 60 and 90 minutes, respectively. Using atomic force microscopy, UV-Vis spectrometry and an electrometer the film characterizations were performed. MLG flakes about $13 \mathrm{~nm}$ thick, $10 \mathrm{~m} \mu^{2}$ of area were observed using AFM. In films obtained with 35 immersions the electrical resistance and the resistance sheet reached $1.8 \mathrm{k} \Omega$ and $1.9 \mathrm{k} \Omega /$ square respectively. The light transmittance reached $10-20 \%$ while films obtained with dispersions treated for 60 and 90 minutes were electrically continuous. Thus, conductive films can be used in chemiresistor sensors, electrochemical and supercapacitors electrodes.
\end{abstract}

Keywords: graphene, thin films, dip coating, conductive films, AFM

\section{Introduction}

Despite being known for long graphene can be classified as a new material because only in 2004 it was first observed and isolated ${ }^{1}$. Studies about graphene have been reported since $1859^{2}$ until Geim and Novoselov experimentally isolated it using micromecanic cleavage method ${ }^{1,3}$. Its excellent properties allow a lot of applications in different fields ${ }^{3}$. The range of applications are related to its high mechanical resistance and low density, an alternative to composite with inferior properties, e.g. lower hardness and higher density. Its great thermal and electrical conductivity can be considered as a silicon substitute ${ }^{2,4}$. The low light absorption and electrical properties may allow its use in touch screen devices, ${ }^{2,5-7}$ biotechnology applications and others ${ }^{8}$.

Graphene is a carbon planar monolayer ordered in hexagonal structure ${ }^{3,8}$ with carbon-carbon chemical bond of $0.142 \mathrm{~nm}^{9}$. Its crystalline structure is a Bravais network with two atoms per unitary cell'. The graphene obtaining can be achieved by micromechanical cleavage, consisting of remotion of the top carbon planes using scotch tape and later transferred to some substrate ${ }^{2,9}$. Another technique is chemical micro exfoliation that consists of the insertion of certain reagents between graphene layers to promote the break of Van der Waals bonds and consequent layer exfoliation ${ }^{8,10,11}$. This effect can be increased by ultrasonic bath treatment. Chemical vapor deposition method applied directly onto a solid substrate, primarily coated with a catalytic film, is another possibility ${ }^{4}$.

Transparent conductive films have been used as an element in new electronic devices ${ }^{5,12}$ such as touch screen, organic light emission device and solar cell ${ }^{13,14}$. For these

*e-mail:rogelamo@gmail.com application its low sheet resistance and a high optical transmittance are essential. The most used material with the above characteristics has been indium tin oxide (ITO), but its high cost of production and mechanical fragility do not allow its use in flexible screens ${ }^{5,12,13}$. Thus, new alternatives have been searched to replace ITO. Graphene has also raised great interest in the electric renewable energy area because it can be used in supercapacitor electrodes ${ }^{15-19}$.

The multilayer graphene ${ }^{20}$ is an alternative due to its low electric resistance, good optical transmittance and high mechanical resistance ${ }^{13}$ useful for flexible screen devices. One graphene layer has $97.7 \%$ light transmittance in $550 \mathrm{~nm}$ (the middle of optical spectra) 2,14,21 $^{2}$ and resistance sheet ranging from $10^{3}$ to $10^{10} \mathrm{Ohms}$ per square ${ }^{6}$. In order to obtain graphene thin film from a dispersion onto any kind of substrates, dip-coating technique has proved to be an interesting method ${ }^{22}$. The advantage of this technique is the obtainment of homogeneous films at low cost with few procedures ${ }^{22-24}$. It has been long used in the industry and still relevant for the production of several ceramic products, as well as galvanizing and painting processes ${ }^{25}$. Woks in the literature have shown dip-coating as a viable alternative to deposit graphene in transparent and flexible substrates, potentially useful in optoelectronic devices ${ }^{26-28}$.

This work presents multilayer graphene (MLG) obtained through low cost dip-coating technique developed with a microcontroller in our laboratory. This reports discusses MLGs obtainment from natural graphite flakes and the its cleavage using ultrasonic process. The study of MLG deposition is related to the ultrasound treatment time and number of immersions in dispersions. The focus was to get conductive films onto commercial glasses. UV-Vis 
transmittance spectroscopy, morphology of MLG flakes with atomic force and optical microscopies were studied.

\section{Experimental}

\subsection{Construction of a low cost automated dip coating system}

A low cost system was entirely projected and constructed in our lab with commercial electronic parts bought in ordinary stores. A software was developed in $\mathrm{C}$ language to get thin film depositions by dip coating and/or self-assembly technique. Compared to other commercial dip coating devices, our equipment cost a fraction of their usual price, allowed depositions in various incidence angles and new parameters can be still inserted in its routine since the program was written in our own lab. This device can control automatically all depositions parameters such as: substrate immersion and removal speeds, the immersion time, number of immersion cycles and substrate position inside and outside the solutions/dispersion. Figure 1 shows the block diagram used in the project and a picture of the mechanical apparatus with the stepper motor and substrate holder that can handle six samples simultaneously.

In Figure 1 the dip coating procedure is presented in four steps. The first step is the immersion speed parameter of the sample holder. The control of the immersion speed is essential to prevent the substrate blade from removing the graphene on the dispersion surface. The second step is the immersion time of the substrate inside the dispersion (or solution). In this step, some graphene adheres on the substrate. The third step is the substrate removal speed which is a parameter to control the graphene deposits present on the top layer of the MLG dispersion. In the fourth step, another important parameter of control is the drying time of the sample. After this drying time, this four-step cycle is restarted and performed as many times as necessary.

\subsection{Multilayer graphene obtaining}

To get multilayer graphene, a dispersion was prepared with $6 \mathrm{mg}$ of expanded graphite donated by Nacional de Grafite Company, dispersed in $30 \mathrm{ml}$ isopropyl alcohol and sorted out in three equal parts. Each part was treated in ultrasonic bath for 30 minutes (dispersion A), 60 minutes (dispersion B) and 90 minutes (dispersion C) in order to exfoliate the graphitic layer to generate multilayer graphene (MLG). The three dispersions obtained were used in dip coating system to deposit MLG in glass slices (10 x 25,4 mm).

\subsection{Glass substrates cleaning}

Solidor Co. $1 \mathrm{~mm}$ thick microscope slides were cut in $10 \times 25.4 \mathrm{~mm}$ size to be used as transparent substrate to MLG deposits. Pure water with detergent at $80^{\circ} \mathrm{C}$ was used to wash the glass slides for 40 minutes and later rinsed with pure water exhaustively. The next step consists of cleaning the substrates in isopropanol at $50^{\circ} \mathrm{C}$ for 20 minutes, followed by acetone at $50^{\circ} \mathrm{C}$ for 20 minutes and washed in pure water again. The last step was the treatment of the glass slides in a $\mathrm{H} 2 \mathrm{SO} 4 / \mathrm{H} 2 \mathrm{O} 2(7: 3)$ bath for $1 \mathrm{~h}$ at $80^{\circ} \mathrm{C}$. Finally, the substrates received an ultrasonic bath during 30 minutes and then were washed exhaustively with pure water.

\subsection{Multilayer graphene depositions}

Using glass substrates treated with the above procedures, deposition cycles (insertion and removal from the MLG dispersion) were programmed in order to get an MLG thin film onto the substrate. To study the influence of the number


Figure 1. Dip coating mechanical device picture and block diagram of the routine for the process. 
of insertions and removals on the electrical resistance, 10 , $15,20,25,30$ and 35 cycles were used with the dispersions $\mathrm{A}, \mathrm{B}$ and $\mathrm{C}$. The immersion and removal speeds were 0.81 and 0.29 centimeter/minute, respectively. The time inside and outside the dispersion was 1 minute for all samples.

\subsection{Samples characterization}

The electrical resistance was measured with two terminal methods. Ten measurements of voltage and current in different places of the surface were made in each sample in order to obtain a medium resistance and its standard deviations. The electrical resistance expressed in $\mathrm{K} \Omega$ is in Table 1. In films with a bigger coating area, four terminals and Van der Pauw method were employed to estimate the sheet resistance expressed as $\Omega /$ square. Only in continuous films it was possible to obtain an electrical response (finite resistance) in order to measure the electrical resistance. The measures were carried out using Keithley Electrometer model 2635A connected to the samples with Signatone Co. tungsten tips. The distance between tips was $1 \mathrm{~mm}$ in all measures and both methods (two or four terminals).

The transmittance in the UV-Visible ranged from 190 to $1100 \mathrm{~nm}$ performed in the FEMTO model CIRRUS 80. A clean glass substrate was used as a reference to this measure, since the $\%$ of light transmitted $(T(\%))$ was calculed by $T(\%)=\left(I / I_{O}\right) x 100$, where $I$ and $I_{O}$ refer to the intensity values transmitted by the sample and clean substrate, respectively. Figure 2 shows a graph with the percentage of light transmitted $T(\%)$ at $550 \mathrm{~nm}$ according to the number of immersions for all samples. These values represent an average of the samples transmittance and used to compare the effect of ultrasound time and number of immersions on the surface

Table 1. Electrical resistance (K $\Omega$ ) obtained by two terminals method.

\begin{tabular}{cccc}
\hline Immersions & Dispersion A & Dispersion B & Dispersion C \\
\hline 15 & - & $148.6 \pm 76.0$ & $40.4 \pm 13.0$ \\
20 & - & $25.2 \pm 2.4$ & - \\
25 & $3.6 \pm 1.7$ & $165.1 \pm 19.0$ & $3.4 \pm 0.6$ \\
30 & - & $3.3 \pm 0.6$ & $4.8 \pm 1.6$ \\
35 & $1.5 \pm 0.2$ & $1.8 \pm 0.4$ & $2.1 \pm 0.3$ \\
\hline
\end{tabular}

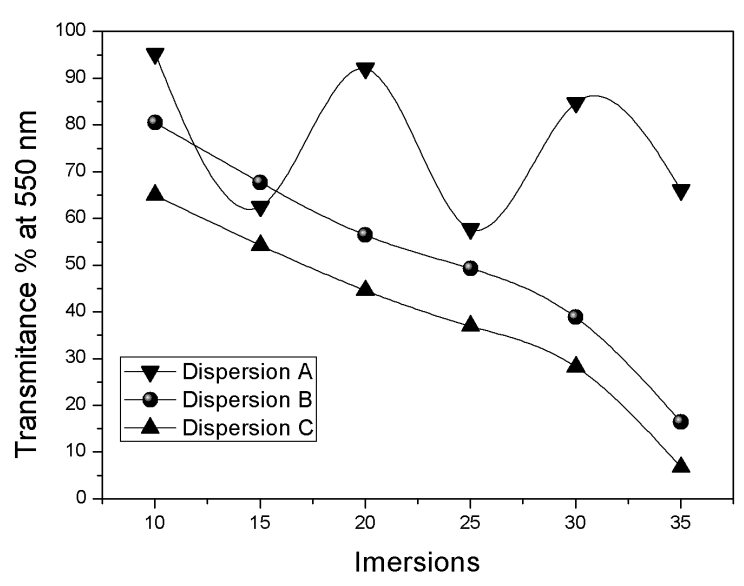

Figure 2. Percentage of light transmitted in $550 \mathrm{~nm}$ to (A), (B) and (C) dispersions as a function of immersions. The lines connecting the experimental points are guide for eyes. covering. This wavelength $(550 \mathrm{~nm})$ has been cited in the literature because of its technological importance to obtain good electrical conductors with high optical transmittance in the middle of visible spectrum ${ }^{29}$.

The surface images were obtained through Zeiss model Axiolab optical microscopy with Achroplan objective (40x zoom and 0.65 of numeric aperture) in transmitted light mode. The pictures were obtained with a Leica model DFC300FX camera directly coupled to the microscopy. To insert the scale in the images the ImageJ v1.49o (Open Source) software was applied.

The MLG flakes morphology and thickness estimates were carried out using Shimadzu atomic force microscopy model SPM9700 in the dynamic mode. The software Shimadzu Manager V.4.01 was used to evaluate the thickness and morphology.

\section{Results and Discussion}

Through our dip coating system and the deposition procedures previously described, 18 samples were obtained onto glass substrates. Figure 3 shows the effect of the exfoliation time in ultrasound on the amount of film formation. The three images in Figure 3 were obtained after 30 immersions with dispersions A, B and C. The dispersion A sample showed the lowest number of coating compared to dispersions $\mathrm{B}$ and $\mathrm{C}$.

A carefully analysis of Figure 2 showed no tendency of gain or loss of material on the glass surface of films obtained with dispersion A. The MLG obtained with dispersion A did not present good adsorb properties on glass. On the other hand, dispersion $\mathrm{B}$ and $\mathrm{C}$ showed a well-defined behavior of light transmitted according to number of immersions. Furthermore, films obtained with dispersion $\mathrm{C}$ are more absorbent than films of dispersion B. Comparing Figures 2 and 3, dispersion $\mathrm{C}$ showed best MLG recovering.

Our results indicate a lower light transmitted reaching 10 and $20 \%$ compared with those related to depositions of non-oxidized graphene flakes, ${ }^{30}$ graphene oxide and graphene oxide with nanowires and nanofibers ${ }^{12,13,29}$ to which transmittances in the range $55-75 \%$ were reported.

To get MLG morphological characterization an AFM equipment in dynamic mode was used for a flake mapping. Samples with 10 immersions were selected because their spaced and isolated flakes provided a better AFM mapping.

AFM images showed a thickness decrease of the flake related to the ultrasound time treatment, an evidence of decrease graphene planes in the flakes. Flakes present in dispersion A sample reached $2 \mu \mathrm{m}$ or more, above the nanometric scale of observation. Through several flakes mapping from dispersion $\mathrm{B}$ and $\mathrm{C}$, it was possible to estimate the thickness of about $16.6 \pm 7.3 \mathrm{~nm}$ and $13.6 \pm 5.2 \mathrm{~nm}$, respectively. No thickness difference could be found with dispersions B and $\mathrm{C}$ samples. Figures 4 and 5 present the mapping of two flakes analyzed in dynamic mode of the AFM and the samples thickness profile was obtained with Shimadzu software.

Considering $0.5 \mathrm{~nm}$ as a reference thickness value to one isolated graphene layer, ${ }^{31}$ it was possible to estimate an average number around 26 to 32 graphene layers in our samples, different from the results obtained by Yun et al. ${ }^{13}$ that reported $40 \mathrm{~nm}$ to their graphene oxide/silver nanowire 


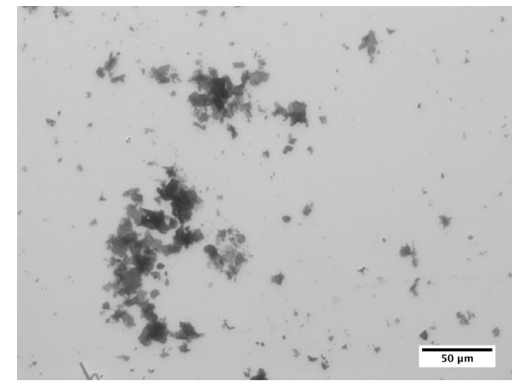

(a)

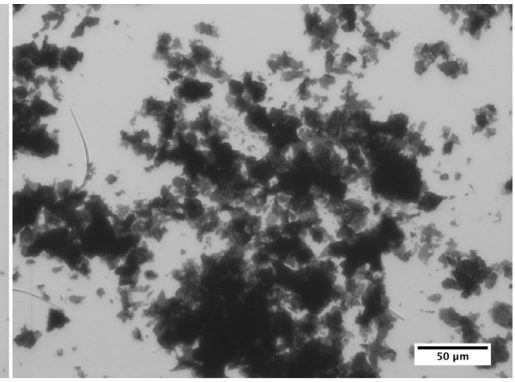

(b)

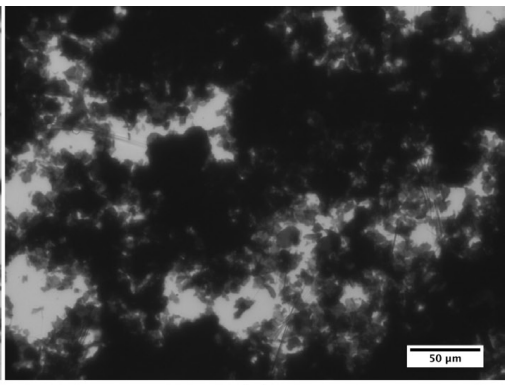

(c)

Figure 3. Optical images obtained for samples treated in ultrassound during 30 minutes (a), 60 minutes (b) and 90 minutes (c) in 30 immersions. The optical zoom is about 40x in transmitted light mode.

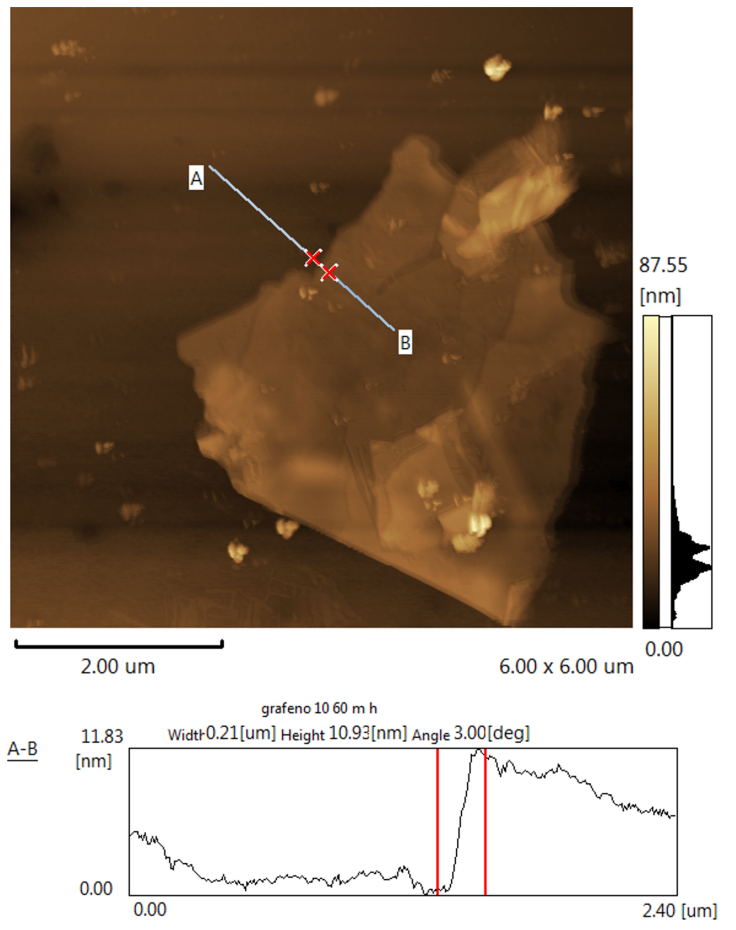

Figure 4. AFM mapping and thickness profile of MLG flake obtained with dispersion $\mathrm{B}$, treated for 60 minutes in ultrasound and 10 immersions. The red crosses situated over the line A-B in micrograph represents the red lines in the surface profile (below micrograph) and were used to determine the graphene flake height.

hybrids films obtained with dip coating method. Since it is not possible to consider the deposit above 10 layers as graphene $^{3}$ our films are multilayer graphene deposits ${ }^{20}$. Another interesting result observed in AFM images (Figures 4 and 5) is the great area of the flakes in the films $\left(\sim 5\right.$ to $\left.10 \mu \mathrm{m}^{2}\right)$.

Although a little fluctuation in the resistance value of the film from dispersion $\mathrm{C}$ with 30 immersions, there is a coherent decrease in the resistance values as a function of the number of immersion, reaching a minor value in the sample with 35 immersions (Table 1). It is possible to conclude that dispersions obtained at 60 and 90 minutes (B and $\mathrm{C}$ ) have a tendency to form a conductive film as it shows a important decrease in the electrical resistance, from 148.6 and $40.4 \mathrm{k} \Omega$ to 1.8 and $2.1 \mathrm{k} \Omega$ for dispersion B and $\mathrm{C}$, respectively. Considering the standard deviation in



Figure 5. AFM mapping and thickness profile of MLG flake obtained with dispersion $\mathrm{C}$, treated for 90 minutes in ultrasound and 10 immersions. The red crosses situated over the line A-B in micrograph represents the red lines in the surface profile (below micrograph) and were used to determine the graphene flake height.

the results for samples obtained with 35 immersion for B and $\mathrm{C}$ dispersions, the electric resistance tends to the same value. Through a careful analysis of Figure 3 , it is possible to see that the spaces not filled by MLGs are smaller than the distance $(1 \mathrm{~mm})$ between probe tips used to get electric resistance. This implies an electric homogeneity in millimeter scale in the films deposited with dispersion B and C.

The sheet resistance obtained by Van der Pauw reached $4700 \Omega /$ square and $1880 \Omega$ /square from samples obtained with 35 immersions in dispersion $\mathrm{B}$ and $\mathrm{C}$ respectively. These values are higher than $200 \Omega /$ square obtained by Yun et al. ${ }^{13}$ with graphene hybrid films as well as those commercial ITO $^{13}$. Nevertheless Yun et al. ${ }^{13}$ obtained this resistance at around $86 \%$ of light transmitted, while our results are around $10 \%$. Park et al. ${ }^{30}$ deposited graphene films chemically exfoliated 


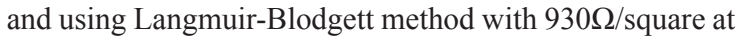
$75 \%$ of transmittance.

Despite the presence of more MLG flakes in the surface confirmed by optical images (Figure 3 ) and low light transmitted (Figure 2), the sample obtained with dispersion $\mathrm{C}$ with 35 immersions probably reached a limit value for the electric resistance and any excess of MLG flakes does not act in the decrease of this electric resistance value. The contact resistance is another parameter that can be influencing the electric resistance obtained. As the graphene flakes are formed by overlapping, a weak contact takes place between them and does not contribute to the reduction of samples resistance in the same proportion of optical transmittance decrease. Furthermore the tungsten tips can damage the film during the measure process, introducing additional contact resistance on the results. The contact resistance can be improved by an annealing treatment or using some nanoparticle or nanofiber to connect electrically and mechanically the graphene flakes as Yun et al. ${ }^{13}$ did. However, this is not the scope of this work and will be investigated in a future work. In order to increase the optical transmittance additional experimental procedures is necessary in our samples. An alternative consists to use some clean treatment to remove overlapping flakes as well as those not adhered to the surface without removing the flakes in contact with substrate. The flakes not adhered and the ones overlapped on glass surface can act as a barrier to the transmitted light and contribute to the low transparence here observed.

To obtain the graphene flakes we used few procedures and chemical reagents compared with recent works in the literature. Another advantage is the use of natural graphite

\section{References}

1. Novoselov KS, Geim AK, Morozov SV, Jiang D, Zhang Y, Dubonos SV, et al. Electric field effect in atomically thin carbon films. Science. 2004; 306(5696):666-669. http://dx.doi. org/10.1126/science.1102896. PMid:15499015.

2. Novoselov KS. Nobel lecture: graphene: materials in the flatland. Reviews of Modern Physics. 2011; 83(3):837-849. http://dx.doi. org/10.1103/RevModPhys.83.837. PMid:21732505.

3. Geim AK and Novoselov KS. The rise of graphene. Nature Materials. 2007; 6(3):183-191. http://dx.doi.org/10.1038/ nmat1849. PMid:17330084.

4. Jesus K.A., Freire E. and Guimarães M.J. Grafeno: aplicações e tendências tecnológicas. Revista de Química Industrial. 2012; 737:14-19.

5. Wang SJ, Geng Y, Zheng Q and Kim J-K. Fabrication of highly conducting and transparent graphene films. Carbon. 2010; 48(6):1815-1823. http://dx.doi.org/10.1016/j.carbon.2010.01.027.

6. Yanwu Z, Cai W, Piner RD, Velamakanni A and Ruoff RS. Transparent self-assembled films of reduced graphene oxide platelets. Applied Physics Letters. 2009; 95(1):103-104.

7. Yang Z, Gao R, Hu N, Chai J, Cheng Y, Zhang L, et al. The prospective two-dimensional graphene nanosheets: preparation, functionalization, and applications. Nano-Micro Let. 2012; 4(1):1-9. http://dx.doi.org/10.1007/BF03353684.

8. Mohammad $\mathrm{H}$ and Paransa A. Graphene: synthesis and applications in biotechnology - a review. World Applied Programming. 2012; 2(6):377-388. without oxidation to obtain the exfoliation. Although the samples studied here did not exhibit high transparency in visible region, a low resistance sheet was observed

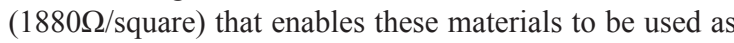
catalysis electrodes, chemiresistor sensors, as well as in electrochemical capacitors which need a great area/volume ratio found in graphene structures.

\section{Conclusions}

This work presents the development of dip coating equipment to deposit a large range of thin films at low cost. The deposition setup is managed by a microcontroller and uses several parameters to control the depositions from dispersions or solutions.

MLG with few graphene layers and micrometric area were obtained by exfoliation of graphite in ultrasonic bath at different times of treatment. Films were deposited using MLG dispersion treated for 30,60 and 90 minutes of ultrasound. Films electrically homogenous with relatively low electric resistance and low percentage of light transmitted were obtained using 60 and 90-minutes dispersions, but 30-minutes dispersion did not show electrical homogeneity. The films herein obtained can be used in chemical and biological sensors, electrochemical applications, flexible conductors substrates and electrochemical capacitors.

\section{Acknowledgements}

The authors thank CNPq and FAPEMIG for the financial support and Nacional de Grafite Co. to the graphite donated to ours experiments.

9. Caldeira BJ. Transporte elétrico em nanoestrutura de grafeno: influência da funcionalização, da geometria e da dopagem do substrato. [Thesis]. Belo Horizonte: Universidade Federal de Minas Gerais; 2011.

10. Geim AK. Graphene: status and prospects. Science. 2009; 324(5934):1530-1534. http://dx.doi.org/10.1126/science.1158877. PMid:19541989.

11. Dreyer DR, Park S, Bielawski CW and Ruoff RS. The chemistry of graphene oxide. Chemical Society Reviews. 2010; 39(1):228240. http://dx.doi.org/10.1039/B917103G. PMid:20023850.

12. Huang YL, Baji A, Tien HW, Yang YK, Yang SY, Wu SY, et al. Self-assembly of silver-graphene hybrid on electrospun polyurethane nanofibers as flexible transparent conductive thin films. Carbon. 2012; 50(10):3473-3481. http://dx.doi. org/10.1016/j.carbon.2012.03.013.

13. Yun YS, Kim DH, Kim B, Park HH and Jin H-J. Transparent conducting films based on graphene oxide/silver nanowire hybrids with high flexibility. Synthetic Metals. 2012; 162(1516):1364-1368. http://dx.doi.org/10.1016/j.synthmet.2012.05.026.

14. Naito K, Yoshinaga N, Tsutsumi E and Akasaka Y. Transparent conducting film composed of graphene and silver nanowire stacked layers. Synthetic Metals. 2013; 175(1):42-46. http:// dx.doi.org/10.1016/j.synthmet.2013.04.025.

15. Simon P and Gogotsi Y. Materials for electrochemical capacitors. Nature Materials. 2008; 7(11):845-854. http://dx.doi.org/10.1038/ nmat2297. PMid:18956000.

16. Yoo JJ, Balakrishnan K, Huang J, Meunier V, Sumpter BG, Srivastava A, et al. Ultrathin planar graphene supercapacitors. 
Nano Letters. 2011; 11(4):1423-1427. http://dx.doi.org/10.1021/ nl200225j. PMid:21381713.

17. Wu ZS, Parvez K, Feng X and Müllen K. Graphene-based in-plane micro-supercapacitors with high power and energy densities. Nature Communications. 2013; 4(2487):2487. PMid:24042088

18. Liu L, Niu Z, Zhang L, Zhou W, Chen X and Xie S. Nanostructured graphene composite papers for highly flexible and foldable supercapacitors. Advanced Materials. 2014; 26(28):4855-4862. http://dx.doi.org/10.1002/adma.201401513. PMid:24838633.

19. Peng X, Peng L, Wu C and Xie Y. Two dimensional nanomaterials for flexible supercapacitors. Chemical Society Reviews. 2014; 43(10):3303-3323. http://dx.doi.org/10.1039/c3cs60407a. PMid:24614864.

20. Rouxinol FP, Gelamo RV, Amici RG, Vaz AR, Moshkalev SA. Low contact resistivity and strain in suspended multilayer graphene. Applied Physics Letters. 2010; 97(25):253104-1253104-3. http://dx.doi.org/10.1063/1.3528354.

21. Allen MJ, Tung VC and Kaner RB. Honeycomb carbon: a review of graphene. Chemical Reviews. 2010; 110(1):132-145. http://dx.doi.org/10.1021/cr900070d. PMid:19610631.

22. Simêncio ECA. Preparação e caracterização de filmes finos sol-gel de Nb2O5:Zr. [Dissertation]. São Carlos: Universidade de São Paulo; 2009.

23. Song YI, Kim GY, Choi HK, Jeong HJ, Kim KK, Yang CM, et al. Fabrication of carbon nanotube field emitters using a dip-coating method. Chemical Vapor Deposition. 2006; 12(6):375-379. http://dx.doi.org/10.1002/cvde.200506442.

24. Melo LO. Preparação e caracterização de filmes finos sol-gel de Nb2O5 dopados com $\mathrm{Li}+$ visando possível aplicação em arquitetura. [Dissertation]. São Carlos: Universidade de São Paulo; 2001.
25. Sánchez FAL. Produção por dip coating e caracterização de revestimentos compósitos de poliuretano/alumina sobre fibras de poliamida 6. [Dissertation]. Porto Alegre: Universidade Federal do Rio Grande do Sul; 2009.

26. Meenakshi P, Karthick R, Selvaraj M and Ramu S. Investigations on reduced graphene oxide film embedded with silver nanowire as a transparent conducting eletrode. Solar Energy Materials and Solar Cells. 2014; 128:264-269. http://dx.doi.org/10.1016/j. solmat.2014.05.013.

27. Saleh ZS, Anyi CL, Haris H, et al. Q-switched Erbium-doped fiber laser using multi-layer graphene oxide based saturable absorber. Optoeletronics an Advanced Materials-Rapid Communications. 2014; 8(7-8):626-630.

28. Oh JS, Kim KN and Yeom GY. Graphene doping methods and device applications. Journal of Nanoscience and Nanotechnology. 2014; 14(2):1120-1133. http://dx.doi.org/10.1166/jnn.2014.9118. PMid:24749416.

29. Tien HW, Hsiao ST, Liao WH, Yu YH, Lin FC, Wang YS, et al. Using self-assembly to prepare a graphene-silver nanowire hybrid film that is transparent and electrically conductive. Carbon. 2013; 58:198-207. http://dx.doi.org/10.1016/j.carbon.2013.02.051.

30. Park KH, Kim BH, Song SH, Kwon J, Kong BS, Kang K, et al. Exfoliation of non-oxidized graphene flakes for scalable conductive film. Nano Letters. 2012; 12(6):2871-2876. http:// dx.doi.org/10.1021/n13004732. PMid:22616737.

31. Morozov SV, Novoselov KS, Schedin F, Jiang D, Firsov AA and Geim AK. Two-dimensional electron and hole gases at the surface of graphite. Physical Review B: Condensed Matter and Materials Physics. 2005; 72(20):201401. http://dx.doi. org/10.1103/PhysRevB.72.201401. 